\title{
Simultaneous Percutaneous Alcohol Ablation of Pulmonary and Hepatic Hydatid Cysts: "PAIR" in a Pair
}

\author{
Udit Chauhan $^{1}$ Sunil Kumar Puri ${ }^{1} \quad$ Neeraj Jain ${ }^{1} \quad$ Sudheer Pargewar ${ }^{2}$ Vandana Goel ${ }^{1}$ \\ ${ }^{1}$ Department of Radiology, Gobind Ballabh Pant Institute of Post \\ Graduate Medical Education and Research, New Delhi, India \\ 2 Department of Radiology, Institute of Liver and Biliary Sciences, \\ New Delhi, India \\ Address for correspondence Udit Chauhan, MD, DNB, PDCC, \\ Department of Radiology, Gobind Ballabh Pant Institute of Post \\ Graduate Medical Education and Research, New Delhi-110002, India \\ (e-mail: dr.udit.chauhan@gmail.com).
}

J Clin Interv Radiol ISVIR 2017;1:124-129.

\begin{abstract}
Keywords

- pulmonary

- hydatid

- percutaneous

- alcohol ablation

- PAIR

We present a 48-year-old lady with left chest pain and dyspnea of 6 months duration. Computed tomography scan revealed a left-sided cystic lesion in the lung parenchyma abutting the posterior pleura and chest wall along with a similar cystic lesion in the segment IV of the liver. A diagnosis of lung and liver hydatid cysts was made based on the imaging and positive serology. Percutaneous alcohol ablation of the lung and hepatic hydatid cysts was done using catheter drainage (PAIR-D: percutaneous aspiration, instillation of scolicidal agents, reaspiration of contents, and drainage) in the lung cyst and needle-PAIR in the liver lesion. The procedure was well tolerated without any intra- as well as postprocedure complication. The patient is well during 24 months follow-up without any recurrence or morbidity.
\end{abstract}

\section{Introduction}

Hydatid disease is caused by the larval forms of the cestode, Echinococcus granulosus where man is the intermediate host. ${ }^{1}$ Hydatid disease is a zoonotic disease that is known to occur worldwide. ${ }^{2}$ The highest prevalence of human hydatid disease in India has been reported from Andhra Pradesh, Saurashtra, and Tamil Nadu. ${ }^{3}$ Most commonly affected organs are the liver $(\sim 75 \%)$ and lungs $(\sim 15 \%) .{ }^{4}$ It is a major health problem with significant morbidity if left untreated. Lungs act as a second filter for the hydatid cysts in the human body and thus the second most common involved site. ${ }^{4}$ Various treatment options exist, ranging from pharmacological management to surgical resection. For pulmonary hydatid cysts, surgery is considered as first-line treatment with lung-preserving surgeries such as enucleation, pericystectomy, cystectomy or cystostomy with or without capitonnage being the options. ${ }^{5,6}$ Due to significant morbidity associated with surgery, there is an upsurge in minimally invasive surgical techniques such as thoracoscopic removal of the cysts located subpleurally. ${ }^{7}$ These techniques have been manipulated as an adjunct to the medical treatment. ${ }^{7,8}$
There are a few reports in the indexed literature stating the feasibility of thoracoscopic instillation of scolicidal agents especially hypertonic saline. ${ }^{7}$ Also, there are also stray anecdotal reports of percutaneous injection of scolicidal agents into the pulmonary hydatid cyst.

However, PAIR technique has been extensively used and is now considered as a well-established treatment option in appropriate cases of hepatic hydatid cysts. Encouraged by the results of PAIR in hepatic cysts and coupled with the fact that the pulmonary cyst in our case was abutting the chest wall and had a very easy access, we attempted PAIR-D (percutaneous aspiration, instillation of scolicidal agents, reaspiration of contents, and drainage) in the pulmonary cyst and a needlePAIR in hepatic cyst in the same sitting. A written informed consent from the patient and clearance from the institutional review board was obtained before the procedure.

\section{Case Report}

A 48-year-old lady presented with left chest pain and dyspnea of 6 months duration. Abnormal physical findings were confined to the chest, which revealed dampened breath
DOI https://doi.org/ $10.1055 / \mathrm{s}-0037-1602773$. ISSN 0000-0000.
Copyright ( 2017 by Indian Society of Vascular and Interventional Radiology
License terms

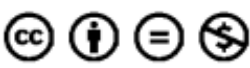




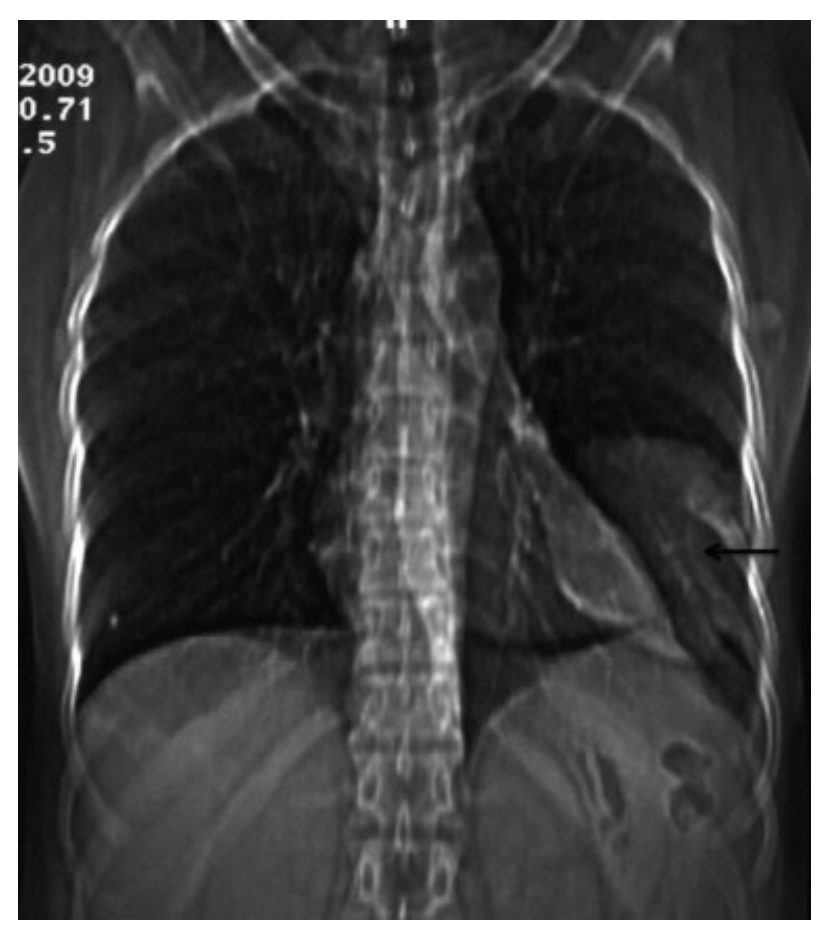

Fig. 1 Chest X-ray showing well-defined radioopaque lesion in the lower zone of the left lung (arrow).

sounds over the left lower chest. Routine blood investigations were unremarkable. Chest X-ray revealed a well-defined oval radioopaque lesion in the lower zone of left lung (- Fig. 1). CT scan revealed a cystic lesion of size $6.5 \times 5.8 \times 6.2 \mathrm{~cm}$ with moderately thick walls in the anterior and lateral basal segments of left lower lobe (-Fig. 2A-C). The lesion was seen abutting the pleura and lateral chest wall with minimal perilesional lung atelectasis. No septae, membranes or solid component were seen inside the cyst. No adjacent pleural reaction/fluid was noted. No other focal lung parenchymal lesion was noted. CT scan of the abdomen revealed a similar cystic lesion of size $5.3 \times 4.9 \times 3.8 \mathrm{~cm}$ in the segment IV of the liver (-Fig. 3A). Sonography of the chest revealed an anechoic cyst with no intracystic contents. Indirect hemagglutination test for hydatid was positive. Contact history with livestock or pet was negative. Based on the above findings a diagnosis of lung and liver hydatid cysts was made. The patient was explained about the disease and treatment options. Subsequently, percutaneous alcohol ablation of the lung and hepatic hydatid cyst was planned using catheter drainage (PAIR-D) for the lung and needlePAIR for the liver lesion because of its smaller size. Approval from the institutional review board was taken before the procedure.

\section{Technical Details}

Patient received oral albendazole, $10 \mathrm{mg} / \mathrm{kg}$ for 2 weeks before and after the procedure. Coagulation profile was normal. The patient fasted overnight. To minimize the possibility of allergic reaction, intravenous promethazine (25 mg) and steroid (hydrocortisone $200 \mathrm{mg}$ ) were adminis- tered 2 hours before the procedure. The procedure was performed using local anesthesia coupled with mild sedation (intravenous pentazocin: $0.3-0.5 \mathrm{mg} / \mathrm{kg}$ and promethazine: $1 \mathrm{mg} / \mathrm{kg}$ ), under monitored anesthesia care and with the availability of all resuscitative measures. Under the sonographic guidance and all aseptic precautions, the lung cyst was punctured at the point of chest wall abutment using single step 8.5F “Universal Curved Drainage Catheter" $(25 \mathrm{~cm}$ length) (COOK Medical, Bloomington, Illinois, United States) with "trochar" technique. We purposefully avoided "Seldinger" technique using needle and wire as it needs to dilate the track which has a risk of anaphylaxis due to spillage of cyst contents. A clear spring fluid was aspirated. Initially, 10 to $20 \mathrm{~mL}$ of cyst contents was aspirated to decompress the cyst and reduce its turgidity. At this stage, the membranes were separated (-Fig. 4A, B). Following catheter insertion, the cyst was evacuated completely. The membranes inside the cavity detached at this point. Effort was made to aspirate as much of the membranes as possible. Around $80 \mathrm{~mL}$ of $95 \%$ weight/volume alcohol, which was approximately two-third of the aspirated volume, was used as the scolicidal agent and was injected slowly through the catheter over a period of 5 to 7 minutes, always keeping in mind not to over distend the cavity. Alcohol was left inside the cavity for 20 to 30 minutes and the posture of the patient was changed every 5 to 7 minutes for proper contact of alcohol with all the cyst walls. After that the entire contents were aspirated leaving behind approximately $5 \mathrm{~mL}$ of fluid, and the catheter was attached to a drainage bag with a water seal (to prevent pneumothorax if any) for external drainage. The duration of the treatment session was 45 minutes. Postprocedure, the fluid drained was examined microscopically and found negative for live protoscolices. A chest X-ray and CT scan of the chest and abdomen were performed after 1 week to check the position of the catheter and the status of the cyst. At 1 week, the cyst had collapsed and was much smaller (-Figs. 3B and $\mathbf{4 B}$ ). The catheter was left in situ till the cyst drained less than $10 \mathrm{~mL}$ fluid per day for 3 consecutive days, after which the catheter was finally removed. The procedure took a total of 8 days from the placement till the removal of the catheter.

Needle-PAIR was done for the hepatic hydatid cyst without insertion of the catheter. The procedure is essentially the same as described above except catheter is not inserted, and alcohol ablation of the cyst is done via the needle in situ. A few technical precautions are to be taken in case of hepatic hydatid: first, the puncture is done traversing a safe and sufficient $(1-1.5 \mathrm{~cm})$ rim of liver parenchyma; second, always check for the biliary pigments using urine dipstick test and in case biliary communication is present, sclerosing agent is not instilled to prevent the development of cholangitis.

The procedure was well tolerated and was uneventful without any intra- as well as postprocedural complication. The patient was subsequently discharged on albendazole. After catheter pull out, follow-up imaging was obtained with chest radiographs only for the lung cyst and ultrasound for the liver cyst at 2 weeks, 4 weeks, 3 months, and then 6 monthly intervals. Noncontrast CT chest and abdomen was 


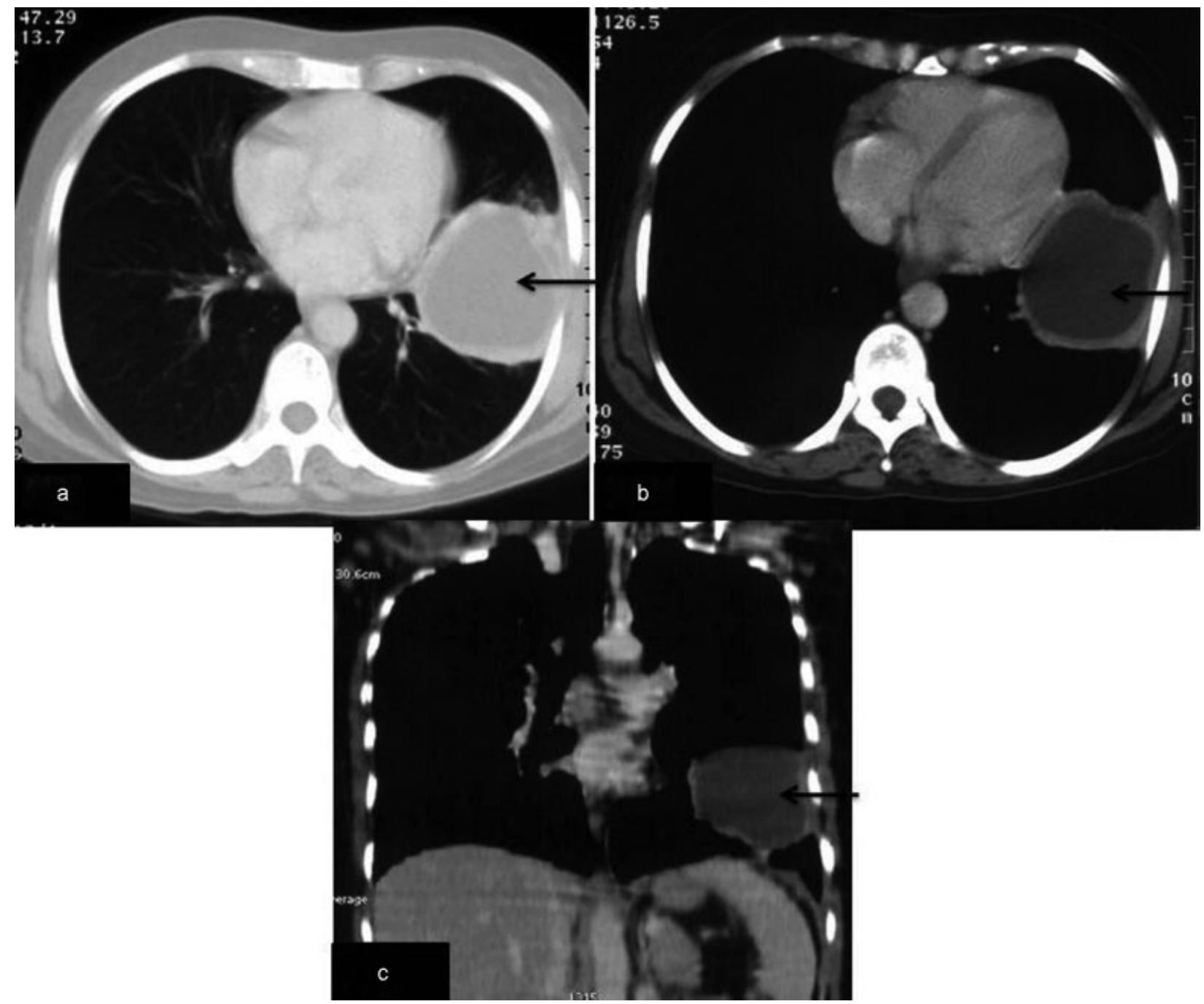

Fig. 2 CECT axial lung window (A) axial mediastinal window (B) and coronal mediastinal window (C) images showing cystic lesion with the moderately thick rim of atelectasis in anterior and lateral basal segments of the lower lobe of the left lung, abutting the pleura and chest wall. CECT, contrast-enhanced computed tomography.

also obtained at 1 year and 2 year intervals. Throughout the follow-up, patient remained asymptomatic. CT scan at 1 year revealed a small collapsed cavity with membranes in the left lower lobe and at 2 years revealed an only small area of subsegmental atelectasis (-Fig. 4C-F). No fluid content was noted at any of follow-up scan. The liver cyst had also collapsed with the suggestion of clumped membranes and absence of fluid (-Fig. 3C) which at 2-year follow-up was reduced to a hypodense lesion of significant small size without any appreciable membranes ( - Fig. 3D). Both the liver as well as the lung cysts showed more than $70 \%$ volume loss suggestive of a good response to ablative treatment.

\section{Discussion}

The liver is the most common site for hydatid disease with right lobe being affected most frequently. Most commonly patients may remain asymptomatic but few experience a dull pain in the right upper abdomen. There may be a presentation with various complications such as rupture into the peritoneal cavity and causing anaphylaxis, rupture into the biliary system, transdiaphragmatic spread into the lung, peritoneal seedling, portal vein involvement, and its thrombosis. Hematogenous spread can also occur into the lungs, spleen, kidneys, bone, and brain. ${ }^{4}$ A large proportion of pulmonary hydatid cysts may remain asymptomatic for a long time until they grow large enough to cause signs and symptoms, such as a nonproductive cough, chest pain, and dyspnea. Complications include rupture, secondary infection, pneumothorax, and suppuration may sometimes precede the diagnosis. ${ }^{3,9}$ Various treatment modalities are available for treating pulmonary hydatid disease including medical and surgical options. Till now the only successful treatment for treating uncomplicated cysts included surgery. Medical treatment with benzimidazoles (mebendazole and albendazole) causes disappearances of cysts in $30 \%$, partial response in 30\%, and no response in remaining $40 \%$ especially if their size is $>4 \mathrm{~cm}$ and with thick walls. ${ }^{7}$ It is indicated in young patients and patients with high risk for surgery. ${ }^{7}$ We used prophylactic albendazole because it has been proven to 

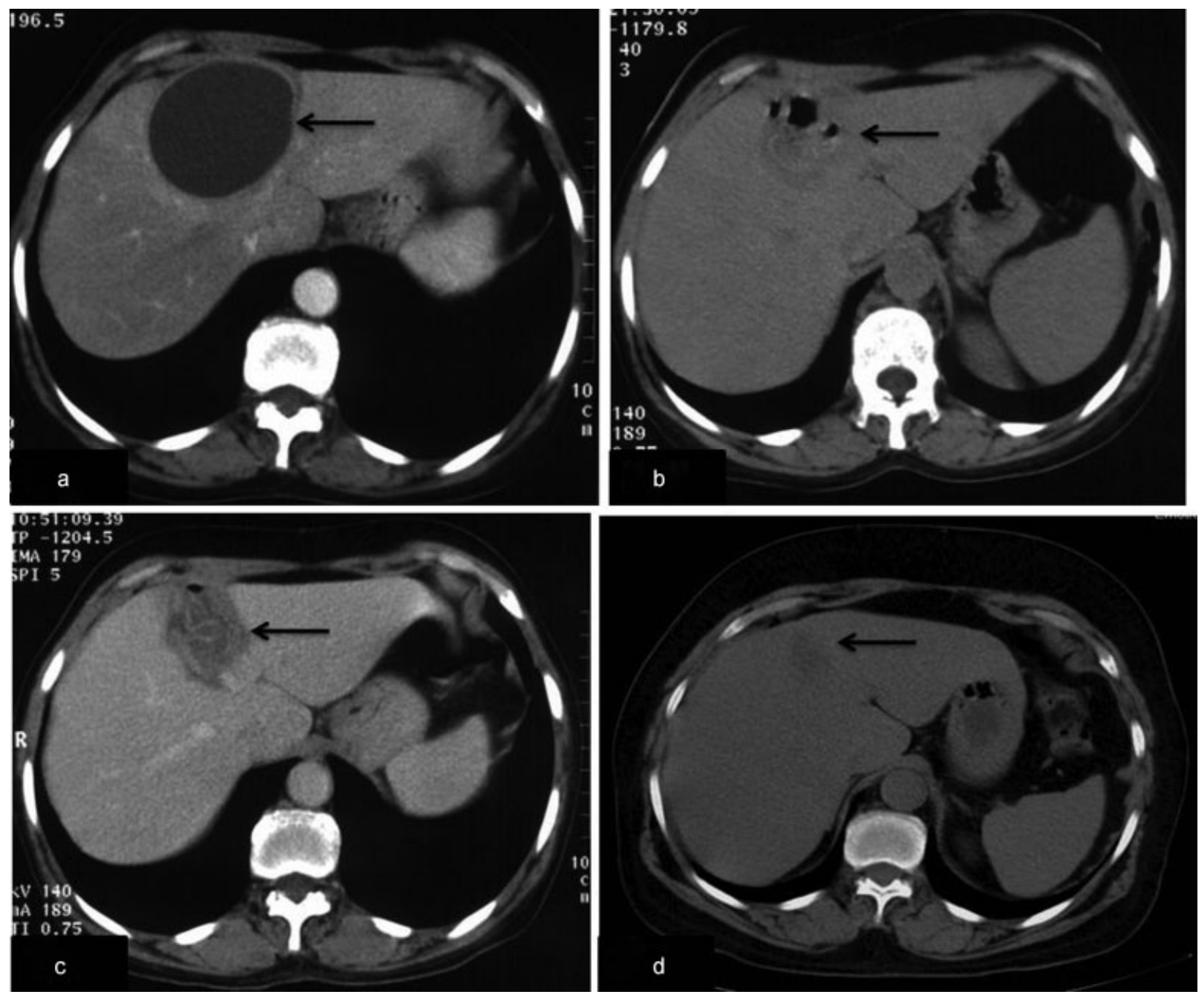

Fig. 3 CECT axial image (A) shows a well-defined hypodense cystic attenuation lesion in segment IV of the liver (arrow). NCCT axial image (B) at 1-week post needle-PAIR displaying regression of size of the cyst with internally detached membranes seen as hyperdense structures. CECT axial image (C) at 1.5 years reveals regression of size of the cyst with detached internal membranes and no significant fluid components. NCCT axial images (D) at 2 years reveals significant regression in size of cyst seen as a subtle hypodense lesion. CECT, contrast-enhanced computed tomography; NCCT, non-contrast computed tomography.

have a protective effect against spillage ${ }^{10}$ and also has an additive positive effect when used along with percutaneous treatment rather than any of these treatments alone. ${ }^{11}$ Due to significant morbidity associated with surgical procedures such as enucleation, pericystectomy, cystectomy or cystostomy with or without capitonnage various minimally invasive procedures have evolved for the treatment of pulmonary hydatid disease. Minimally invasive procedures included video-assisted thoracoscopic surgery, ${ }^{12}$ video thoracoscopy and cystectomy, ${ }^{8}$ medical thoracoscopy with instillation of hypertonic saline ${ }^{7}$ and even percutaneous hypertonic saline irrigation, ${ }^{13}$ etc. Traditionally PAIR/PAIR-D have been used in the treatment of hepatic hydatid disease with very good results. Various scolicidal agents such as hypertonic saline, 95\% alcohol, and betadine have been tried with equally good results. However, there are only anecdotal reports in literature where percutaneous treatment has been performed using hypertonic saline as scolicidal agent for the treatment of pulmonary hydatid cyst. ${ }^{13}$ According to the literature published by Rai S, P et al, it was found that the cases which responded to albendazole in the initial course itself showed regression in size of the cyst and responded well during further cycles leading to complete response while in others there was an incomplete response to the medical therapy. Further, during their study, they found that the results with hypertonic saline as scolicidal agent in PAIR were not encouraging. ${ }^{13}$ Another report was published by Mawhorter et al in which they had treated pulmonary hydatid cyst with percutaneous aspiration and injection of hypertonic saline. However, in their report, they mentioned about aborting the injection of hypertonic saline, after an initial amount of $150 \mathrm{~mL}$, since the patient developed a vigorous cough during injection followed by bronchospasm and fever which got resolved after a week of conservative management. However, later the patient developed pneumonia and hemoptysis which were uncontrollable and ultimately pneumonectomy was done. The resected specimen did not reveal any residual hydatid. ${ }^{14}$ 


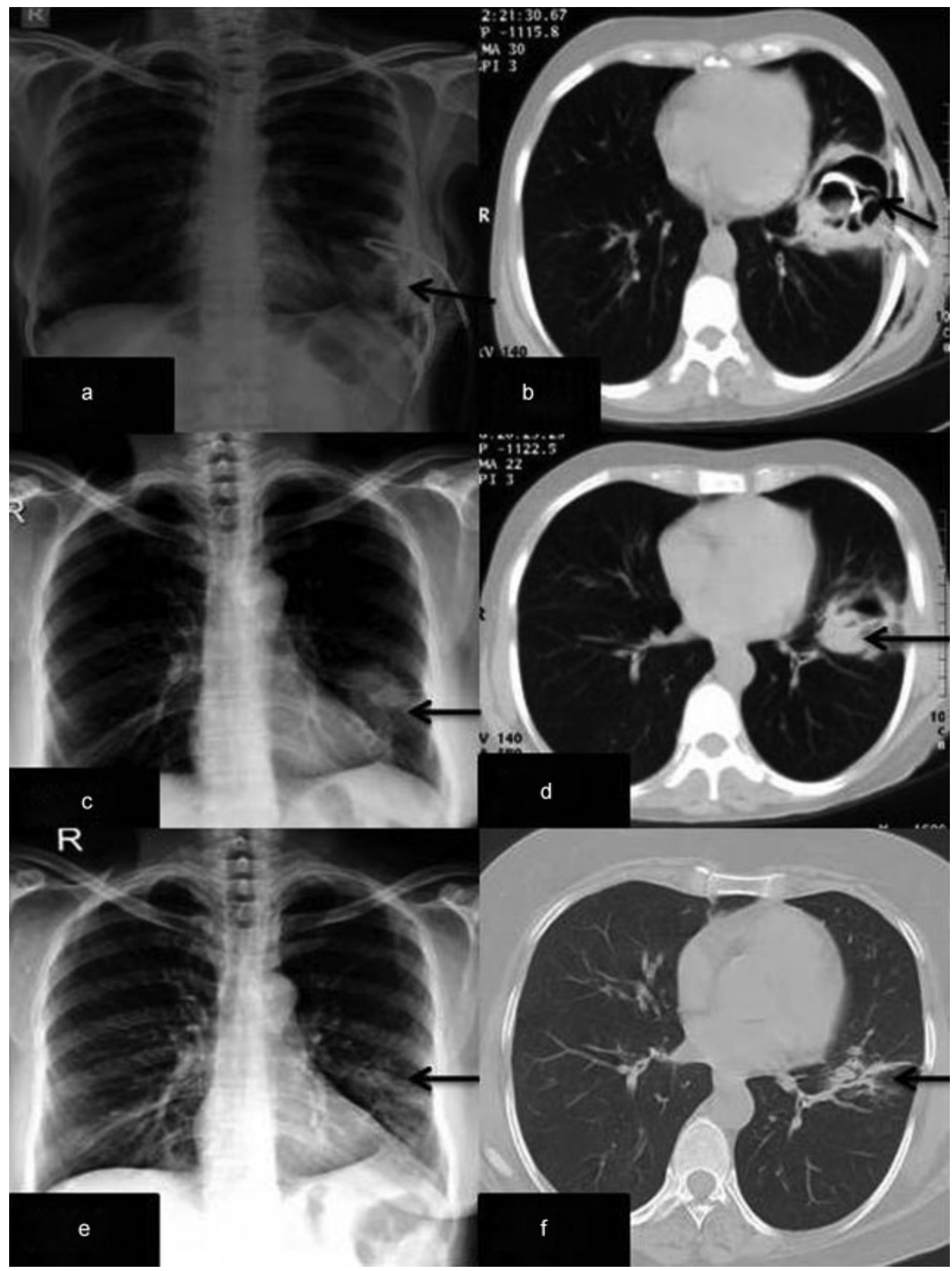

Fig. 4 Chest X-ray (A) and axial lung window (B) at 1 week after the procedure reveal small collapsed cyst in left lower lobe (arrow) with detached internal membranes and drainage catheter in-situ. Follow-up chest X-ray (C) and CT axial image (D) at 1.5 years reveal regression in size of the cavity with hyperdense detached internal membranes. Follow-up chest X-ray (E) and CT axial image (F) at 2 years reveal a small patchy area of fibrotic opacity in left lower zone with diaphragmatic tenting. No residual cavity is visible in these images. Note absence of fluid contents within the cyst on follow-up images suggesting a good response. CT, computed tomography.

Percutaneous ablation of pulmonary hydatid cyst using 95\% alcohol as scolicidal agent with PAIR-D technique is a feasible option especially in patients with peripherally located cysts and in patients with co-morbidities when surgery is not an option. Our case report also highlights the fact that PAIR is an efficacious treatment option for hydatid cysts occurring at the multiple sites and thereby preventing multiple surgeries for the same disease.
Conflict of Interest

The material is not presented elsewhere.

\section{Note}

This study was performed at the Department of Radiology Gobind Ballabh Pant Institute of Post Graduate Medical Education and Research, New Delhi, India. 


\section{References}

1 Richter J, Orhun A, Grüner B, et al. Autochthonous cystic echinococcosis in patients who grew up in Germany. Euro Surveill 2009; 14(22):19229

2 Eckert J, Deplazes P. Biological, epidemiological, and clinical aspects of echinococcosis, a zoonosis of increasing concern. Clin Microbiol Rev 2004;17(01):107-135

3 Topcu S, Kurul IC, Altinok T, Yazici U, Demir A. Giant hydatid cysts of lung and liver. Ann Thorac Surg 2003;75(01):292-294

4 Pedrosa I, Saíz A, Arrazola J, Ferreirós J, Pedrosa CS. Hydatid disease: radiologic and pathologic features and complications. Radiographics 2000;20(03):795-817

5 Aribas OK, Kanat F, Turk E, Kalayci MU. Comparison between pulmonary and hepatopulmonary hydatidosis. Eur J Cardiothorac Surg 2002;21(03):489-496

6 Aribas OK, Kanat F, Gormus N, Turk E. Pleural complications of hydatid disease. J Thorac Cardiovasc Surg 2002;123(03):492-497

7 Lakshmanan PH, Musthafa AM, Suraj K, Ravidran C. Pleuropulmonary hydatid disease treated with thoracoscopic instillation of hypertonic saline. Lung India 2008;25(01):34-37
8 Kerekes L, Takács I, Kollár S, Veres L, Sápy P, Sz Kiss S. Changes in surgical therapy of pulmonary hydatid cysts [in Hungarian]. Magy Seb 2004;57(06):358-363

9 Fatimi SH, Naureen S, Moizuddin SS, et al. Pulmonary hydatidosis: clinical profile and follow up from an endemic region. ANZ J Surg 2007;77(09):749-751

10 Morris DL, Chinnery JB, Hardcastle JD. Can albendazole reduce the risk of implantation of spilled protoscoleces? An animal study. Trans R Soc Trop Med Hyg 1986;80(03):481-484

11 Khuroo MS, Dar MY, Yattoo GN, et al. Percutaneous drainage versus albendazole therapy in hepatic hydatidosis: a prospective, randomized study. Gastroenterology 1993;104(05): 1452-1459

12 Uchikov AP, Shipkov CD, Prisadov G. Treatment of lung hydatidosis by VATS: a preliminary report. Can J Surg 2004;47(05):380-381

13 Rai SP, Panda BN, Ganguly D, Bharadwaj R. Pulmonary hydatid : diagnosis and response to hypertonic saline irrigation and albendazole. Med J Armed Forces India 2005;61(01):9-12

14 Mawhorter S, Temeck B, Chang R, Pass H, Nash T. Nonsurgical therapy for pulmonary hydatid cyst disease. Chest 1997;112(05):1432-1436 